\title{
Enantioselective addition of diethylzinc to benzaldehyde over mesoporous SBA-15 functionalized with chiral proline derivatives
}

\author{
Li-Hsiu Hsiao ${ }^{a}$, Shih-Yuan Chen ${ }^{a}$, Shing-Jong Huang ${ }^{\text {a,b }}$, Shang-Bin Liu ${ }^{b}$, Peng-Huan Chen ${ }^{a}$, \\ Jerry C.-C. Chan ${ }^{a}$, Soofin Cheng ${ }^{a, *}$ \\ a Department of Chemistry, National Taiwan University, Taipei 106, Taiwan \\ ${ }^{\mathrm{b}}$ Institute of Atomic and Molecular Sciences, Academia Sinica, Taipei 106, Taiwan
}

\section{A R T I C L E I N F O}

\section{Article history:}

Received 28 November 2008

Received in revised form 17 February 2009

Accepted 19 February 2009

Available online 3 March 2009

\section{Keywords:}

Asymmetric addition

Proline

Diethylzinc

Benzaldehyde

SBA-15

Mesoporous silica

Xe NMR

\begin{abstract}
A B S T R A C T
Chiral proline derivative was anchored on mesoporous SBA-15 materials by nucleophilic substitution of chloropropyl-functionalized SBA-15 with $(S)-(-)-\alpha, \alpha$-diphenyl-2-pyrrolidinemethanol. Chloropropylfunctionalized SBA-15 prepared by co-condensation of tetraethyl orthosilicate and 3-chloropropyltrimethoxysilane using P123 copolymer as the pore directing agent in strong acidic environment was found to lead to derivatives with chiral centers more homogeneously distributed in the mesopores than that prepared by post-grafting. The resultant materials were very active and selective as catalysts in enantioselective addition of diethylzinc to benzaldehyde to form (S)-1-phenyl-propanol with ca. $66 \%$ of enantiometric excess (e.e.). The e.e. value was further improved to $75 \%$ by adding a small amount of $n-$ BuLi. The reaction rate and the enantioselectivity increased with the accessibility of the chiral proline species in the mesopores and the hydrophobicity around the active sites. Moreover, the catalytic activity was well retained after the used catalyst was regenerated by filtration, washing with $0.2 \mathrm{M} \mathrm{HCl}$ and then neutralization by TMAOH.
\end{abstract}

(c) 2009 Elsevier B.V. All rights reserved.

\section{Introduction}

Asymmetric synthesis is very important in the pharmaceutical industry [1-3]. Secondary alcohols with optical activity are important intermediates for preparing many molecules that mimic natural products and pharmaceutical compounds [4]. One of the well-known routes to prepare secondary alcohols with high optical activity is the asymmetric addition to an aldehyde with dialkyl zinc. The secondary alcohol with higher than 99\% enantiometric excess (e.e.) has been obtained by asymmetric addition of diethyl zinc to benzaldehyde in the presence of a small amount of chiral $\beta$-tertiary amino alcohols as homogeneous catalysts [4-7]. The homogeneous catalysts reported in the literature include the chiral derivatives of camphor, proline, ephedrine, norephedrine, and cinchona alkaloid. The drawbacks of the homogeneous catalytic system are the difficulties involved in separation of the catalyst from the reaction solution, in purification of the products, and in reuse of the catalyst, these processes normally require tedious procedures. As a result, the cost of production is greatly increased. In contrast, the reactions through a heterogeneous catalytic pathway have the advantages of easy

\footnotetext{
* Corresponding author. Tel.: +886 2 33661662; fax: +886 223636359

E-mail address: chem1031@ntu.edu.tw (S. Cheng).
}

purification of the products and easy recycling of the catalyst, making the catalytic processes more environmentally friendly and industrially applicable.

The asymmetric addition to benzaldehyde with diethyl zinc in heterogeneous phase has attracted great attention in recent years [8-13]. Watanabe and Soai [9,10] showed that diphenyl-(1methylpyrrolidin-2-yl) methanol (abbreviated as DPMPM) anchored on polystyrene could catalyze asymmetric ethylation of aldehydes to form the resultant alcohols with $68 \%$ yield and $24 \%$ e.e. in about $77 \mathrm{~h}$. By lengthening the tether between the polymer support and DPMPM with a hexyl group, they could increase the yield and e.e. value to $91 \%$ and $61 \%$, respectively, after $24 \mathrm{~h}$ reaction. However, the thermal stability and swelling of the polymer matrix in solvents may seriously hinder the diffusion of reactants, which have been the major concerns for polymer supports [11].

In search of thermally stable and redeemable solid catalysts, chiral $\mathrm{N}$-alkylnorephedrines anchored on alumina and amorphous silica $[12,13]$ have been investigated. However, the reaction rate and enantioselectivity of immobilized homogeneous catalysts are generally much lower than those of the pristine system. That is attributed to that the chiral selective sites may change their conformation or may be hindered when the catalysts are anchored onto the solid supports. Moreover, the loading and the accessibility of the chiral selective catalyst are dependent on the preparation 
method and are limited by the openings of the surfaces. Mesoporous silica materials of large surface areas and tunable pore diameters are good candidates for immobilization of chiral selectivity catalysts [14-19]. Several groups have immobilized $(1 R, 2 S)$-ephedrine on MCM-41-type mesoporous aluminosilicate [20-23]. However, the observed rate $\left(k_{\mathrm{obs}}=0.17 \mathrm{~h}^{-1}\right)$ and the e.e. value $(47 \%)$ in enantioselective addition to benzaldehyde with diethyl zinc were still lower than those of homogeneous catalyst [22]. Moreover, surface silanol groups of MCM-41 were found to catalyze the racemization. Significant increases in the reaction rate and e.e. selectivity were observed when MCM-41 aluminosilicates of larger average pores ( $5.8 \mathrm{~nm}$ vs. $1.7 \mathrm{~nm}$ ) were used as the catalysts [24]. Up to now, the highest catalytic activity (95\% yield) and enantioselectivity (96\% e.e. of (S)-1-phenyl-propanol) in the addition of diethylzinc to benzaldehyde were obtained over Ti(IV)-TADDOL ( $\alpha, \alpha, \alpha^{\prime}, \alpha^{\prime}$-tetraaryl-2,2-dimethyl-1,3-dioxolan-4,5-dimethanol) complex covalently anchored on controlledpore glasses (CPGs) [25]. However, a stoichiometric amount of tetraalkoxytitanium was consumed during the reaction, and refilling of titanium species after every reaction cycle was inevitable.

Although the loading and the accessibility of the catalytic sites are important for the performance of immobilized chiral catalysts, very few papers have addressed these issues. Moreover, most of the chiral selective catalysts reported in the literature were anchored on the mesoporous silica by post-grafting method. It is difficult to achieve a high loading of the organic groups without tampering with the mesopores due to pore blocking [20-26]. In the present study, we focused on the effects of the homogeneity and loading of the chiral selective sites on the catalytic performance. Proline was anchored onto mesoporous SBA-15 materials by nucleophilic substitution of chloropropyl-functionalized SBA-15 with (S)-(-)- $\alpha, \alpha$-diphenyl-2-pyrrolidinemethanol (Scheme 1). The chloropropyl-SBA-15 was prepared via co-condensation of tetraethyl orthosilicate (TEOS) and 3-chloropropyltrimethoxysilane (CPTMS) in order to achieve high loading and homogeneous distribution of the catalytic sites. The catalytic activity and enantioselectivity of the proline functionalized SBA-15 were examined by the asymmetric ethylation of benzaldehyde. Moreover, the influence of hydrophilicity of the silica surfaces was examined by co-functionalizing the mesoporous materials with methyl and phenyl groups, which were also prepared by cocondensation.

\section{Experimental}

\subsection{Chemicals}

All chemicals and solvents of reagent grade were purchased from Janssen Chiminca, Acros, and Aldrich. They were used without further treatments, except for benzaldehyde, which was purified by distillation.

\subsection{Synthesis of 3-chloropropyl-functionalized mesoporous SBA-15} via co-condensation method

Well-ordered 3-chloropropyl-functionalized SBA-15 materials were prepared by one-pot co-condensation method with the aid of $\mathrm{NaCl}[27,28]$. Four gram of pore-directing agent P123 (Aldrich, $M_{\mathrm{n}}=5800$ ) and $13.04 \mathrm{~g}$ of $\mathrm{NaCl}$ (Acros, 99\%) were dissolved in $160 \mathrm{~mL}$ of $2 \mathrm{M} \mathrm{HCl}$ solution. To this solution, ca. $0.041 \mathrm{~mol}$ of TEOS (Acros, 98\%) was pre-hydrolyzed at $35^{\circ} \mathrm{C}$ for $2 \mathrm{~h}$ before adding CPTMS (Acros, 98+\%). The molar reactant compositions in the gel were 0.0168 P123: $(1-x)$ TEOS: $x$ CPTMS: $7.6 \mathrm{HCl}: 185 \mathrm{H}_{2} \mathrm{O}$, where $x$ denotes the CPTMS/(TEOS + CPTMS) molar ratios that vary from $5 \%$ to $20 \%$. The synthesis solution was stirred at $35{ }^{\circ} \mathrm{C}$ for $22 \mathrm{~h}$, followed by a hydrothermal treatment at $90{ }^{\circ} \mathrm{C}$ under static conditions for $24 \mathrm{~h}$. Subsequently, the solid precipitates were obtained by filtration, washing, and drying at $50{ }^{\circ} \mathrm{C}$ overnight. The P123 was removed by ethanol extraction. The resultant samples are hereafter designated as $x \mathrm{Cl}$-SBA- 15 .

In some cases, $1 \mathrm{~g}$ of the ethanol-extracted sample was stirred in $50 \mathrm{~mL}$ toluene at $80^{\circ} \mathrm{C}$ for $14 \mathrm{~h}$ to achieve further removal of the P123 residue. The resultant sample is designated as $x \mathrm{Cl}$-SBA-15-t.

2.3. Synthesis of SBA-15 dual-functionalized with 3-chloropropyl and methyl (or phenyl) groups via co-condensation method

3-Chloropropyl and methyl (or phenyl) dual-functionalized SBA-15 materials were also prepared by the co-condensation method. The procedure was similar to that of 3-chloropropylfunctionalized SBA-15, except that methyltriethoxysilane (MTES, Acros, 98+\%) or phenyltriethoxysilane (PTES, Acros, 98+\%) was also added while CPTMS was introduced into the TEOS prehydrolyzed mixture. The resultant samples are designated as $10 \mathrm{Me}-15 \mathrm{Cl}-\mathrm{SBA}-15$ and $10 \mathrm{ph}-15 \mathrm{Cl}-\mathrm{SBA}-15$, corresponding to the respective molar reactant compositions in the gels of 0.0168P123:0.75TEOS:0.15CPTMS:0.10MTES (or PTES): $7.6 \mathrm{HCl}$ : $185 \mathrm{H}_{2} \mathrm{O}$. The samples were further treated with $50 \mathrm{~mL}$ toluene solution at $80^{\circ} \mathrm{C}$ for $14 \mathrm{~h}$ to remove the residual P123 and are designated as $10 \mathrm{Me}-15 \mathrm{Cl}-\mathrm{SBA}-15-\mathrm{t}$ and 10ph-15Cl-SBA-15-t.

\subsection{Synthesis of 3-chloropropyl-functionalized mesoporous SBA-15 and amorphous $\mathrm{SiO}_{2}$ via grafting method}

For comparison, 3-chloropropyl-functionalized mesoporous SBA-15 and amorphous $\mathrm{SiO}_{2}$ were also prepared via the grafting method. Pure siliceous SBA-15 was synthesized according to the literature reports $[16,17]$. Prior to the grafting procedure, the calcined siliceous SBA-15 or commercial silica gel (Janssen Chimica, silicagel $0.060-0.200 \mathrm{~mm}$, pore diameter ca. $6 \mathrm{~nm}$ ) was
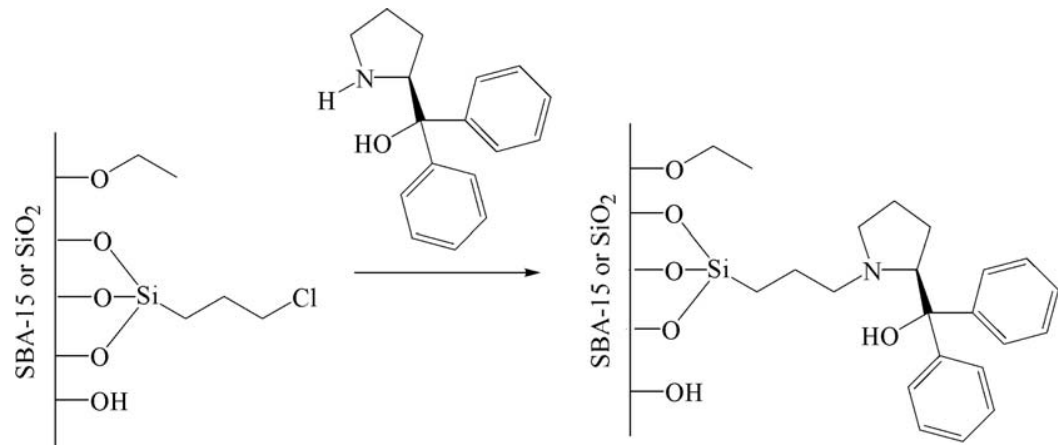

Scheme 1. Anchoring of proline derivative on $\mathrm{SBA}-15$ or $\mathrm{SiO}_{2}$ materials. 
dried at $200{ }^{\circ} \mathrm{C}$ overnight to remove physically adsorbed water. Then, $0.2 \mathrm{~g}$ of the dried silica material was dispersed in $10 \mathrm{~mL}$ of toluene, and $1 \mathrm{~g}$ of CPTMS was added. The molar ratio of CPTMS/ $\mathrm{SiO}_{2}$ was kept at ca. $30 \%$. The solution was then refluxed at $110{ }^{\circ} \mathrm{C}$ for $24 \mathrm{~h}$. Finally, the solid was recovered by filtration; it was washed thoroughly with ethanol, and dried at $100{ }^{\circ} \mathrm{C}$ overnight. The resultant samples are designated as 30Cl-SBA-15-g and 30Cl$\mathrm{SiO}_{2}-\mathrm{g}$, where "g" represents samples prepared by the grafting method.

\subsection{Anchoring of proline derivatives on 3-chloropropyl- functionalized silica materials}

Prior to the substitution experiment, all chloropropyl-functionalized silica materials were dehydrated at $110^{\circ} \mathrm{C}$ overnight. Then, $0.2 \mathrm{~g}$ of the functionalized silica was added into $10 \mathrm{~mL}$ of toluene solution containing various amounts of $(S)-(-)-\alpha, \alpha$-diphenyl-Lprolidinemethanol, and each mixture was refluxed at $110^{\circ} \mathrm{C}$ for $48 \mathrm{~h}$. Finally, the solid materials were filtered, washed with ethanol to remove the remaining organic compounds, and then dried at $100{ }^{\circ} \mathrm{C}$ overnight. These proline-anchored materials are designated as cat- $x \mathrm{Cl}-\mathrm{SiO}_{2}$-g and cat- $x \mathrm{Cl}-\mathrm{SBA}-15-\mathrm{t}$, where "cat" represents the proline-anchored catalysts.

\subsection{Material characterization}

Powdered X-ray diffraction (XRD) patterns were recorded on a Philip X'pert Pro diffractometer with $\mathrm{Cu} \mathrm{K \alpha}$ radiation of $1.5418 \AA$ operated at $40 \mathrm{~mA}$ and $45 \mathrm{kV}$. The pore structures of the materials were analyzed by nitrogen physical sorption at liquid nitrogen temperature $(77 \mathrm{~K})$ using a Micrometerics TriStar 3000 system. Prior to the experiments, the materials were degassed at $120^{\circ} \mathrm{C}$ for more than $8 \mathrm{~h}$ under vacuum $\left(10^{-3}\right.$ Torr $)$. The energy dispersed $\mathrm{X}$ ray (EDX) emission spectra were taken with a Hitachi S-2400 scanning electron microscope. The EDX data were the average results of more than 20 spots. Elemental analyses of $\mathrm{C}, \mathrm{H}, \mathrm{N}$, and $\mathrm{S}$ were performed using a Heraeus VarioEL instrument. FT-IR spectra were recorded using a BOMEM DA 3.02 spectrometer with a resolution of $2 \mathrm{~cm}^{-1}$. Solid-state ${ }^{29} \mathrm{Si},{ }^{13} \mathrm{C}$, and ${ }^{1} \mathrm{H}$ NMR experiments were carried out at frequencies of $59.6,75.5$, and $300.1 \mathrm{MHz}$, respectively, on a Bruker DSX300 NMR spectrometer equipped with a commercial $4 \mathrm{~mm}$ cross-polarization magic-angle-spinning (CP-MAS) probe. The MAS frequencies were set at $6 \mathrm{kHz}$ for all experiments, and the variation was limited to $\pm 3 \mathrm{~Hz}$ with a commercial pneumatic control unit. All ${ }^{29} \mathrm{Si}$ and ${ }^{13} \mathrm{C}$ NMR chemical shifts were externally referenced to tetramethylsilane (TMS).

Variable-temperature (VT) hyperpolarized (HP) ${ }^{129} \mathrm{Xe}$ NMR spectra were acquired on a Bruker AVANCE 300 NMR spectrometer at a Larmor frequency of $83.012 \mathrm{MHz}$ using a single-pulse sequence with a $\pi / 6$ pulse of $5 \mu$ s and a recycle time of $5 \mathrm{~s}$. Prior to each VT-HP ${ }^{129}$ Xe NMR experiment, each powdered sample was first compressed into a self-supported wafer, then, crushed into small pellets, followed by dehydration treatment at $80^{\circ} \mathrm{C}$ under vacuum for $24 \mathrm{~h}$. Details for the fabrication of $\mathrm{HP}{ }^{129} \mathrm{Xe}$ gas by means of a continuous-flow laser-polarized system can be found elsewhere [29]. In brief, a gas mixture $\left(97 \% \mathrm{He}, 1 \% \mathrm{~N}_{2}\right.$ and $2 \%$ natural Xe) controlled at constant pressure ( $4 \mathrm{~atm})$ was introduced into a $\mathrm{Rb}$-containing pumping cell, which was placed in the fringe field (ca. $200 \mathrm{G}$ ) of the superconducting magnet. This mixture was subjected to irradiation of circularly polarized laser light with a wavelength of $794.8 \mathrm{~nm}$. The resulting $\mathrm{HP}{ }^{129} \mathrm{Xe}$ gas (with a polarization of ca. $2 \%$ ) corresponding to a Xe partial pressure of ca. 15.2 Torr was then continuously delivered into the NMR sample chamber under ambient pressure.

\subsection{Asymmetric addition of diethylzinc to benzaldehyde}

The schematic representation of the asymmetric addition of diethylzinc to benzadldehyde over proline-anchored SBA-15 or $\mathrm{SiO}_{2}$ material is shown in Scheme 2. Prior to the experiment, proline-functionalized materials were treated with a $0.2 \mathrm{M}$ methanol solution of tetramethylammonium hydroxide (TMAOH) for $30 \mathrm{~min}$ at room temperature to remove the residual $\mathrm{Cl}^{-}$ions and to neutralize the protonated amine groups in the sample. These steps were followed by washing with a large amount of water and ethanol and drying at $100{ }^{\circ} \mathrm{C}$. All catalytic reactions were carried out in a pyrex flask with a magnetic stirrer. $25 \mathrm{mg}$ of the dried catalyst was first degassed under vacuum, and then cooled down to $0{ }^{\circ} \mathrm{C}$ under $\mathrm{N}_{2}$ atmosphere. To the catalyst, $0.6 \mathrm{~mL}$ of $1 \mathrm{M}$ diethylzinc in $n$-hexane and $25 \mu \mathrm{L}$ benzaldehyde (diethylzinc/ benzaldehyde $=2.4$ ) were added. After various reaction periods, a trace quantity of reaction solution was drawn out and mixed with $0.5 \mathrm{~mL}$ of saturated $\mathrm{NH}_{4} \mathrm{Cl}$ solution to stop the reaction. The procedure was followed by extraction of the products with $3 \mathrm{~mL}$ of ethylacetate. The upper layer solution was collected and filtered; a weighed amount of toluene was added as the internal standard and the resulting mixture was analyzed by a HP6890 GC-MS instrument equipped with a Rt- $\beta$ DEXm column (14\% cyanopropylphenylsiloxane and $86 \%$ dimethylpolysiloxane doped with permethylated beta cyclodextrin) $0.25 \mathrm{~mm}$ in diameter and $30 \mathrm{~m}$ in length. A typical profile of the products separated by GC is shown in Fig. 1. Other than the $(R)$ - and $(S)$-form 1-phenyl-1propanol, a small amount of benzyl alcohol was obtained as the side-product. The selectivity of 1-phenyl-1-propanol and the e.e. preference of $(S)$-1-phenyl-1-propanol were calculated by the following equations based on the molar ratios calibrated from the GC peak areas:

$$
\begin{aligned}
& \text { Selectivity }=\frac{([R]+[S])}{\left([R]+[S]+\left[\mathrm{PhCH}_{2} \mathrm{OH}\right]\right)} \times 100 \% \\
& \% \text { of e.e. }=\frac{([S]-[R])}{([S]+[R])} \times 100 \%
\end{aligned}
$$

\section{Results}

\subsection{Chloropropyl-functionalized SBA-15}

All the chloropropyl-functionalized SBA-15 materials, except the one with the highest CPTMS/TEOS ratio of 0.2 , as well as those co-functionalized with phenyl and methyl groups show three wellresolved X-ray diffraction peaks corresponding to the $\left(\begin{array}{lll}1 & 0 & 0\end{array}\right)$, (1 10$)$, and $(200)$ planes of the 2D-hexagonal p6mm pore structure $[16,17]$. The content and incorporating efficiency (I.E.) of chloropropyl groups in mesoporous SBA-15 were analyzed by

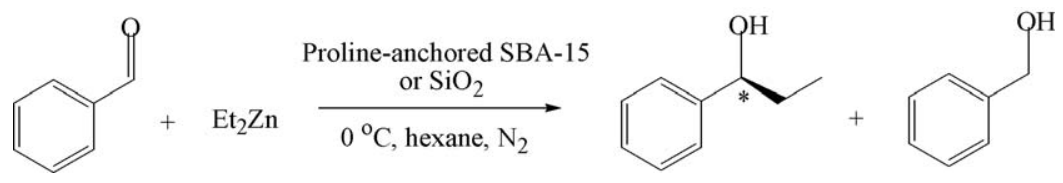

Scheme 2. Asymmetric addition of diethylzinc to benzaldehyde. 


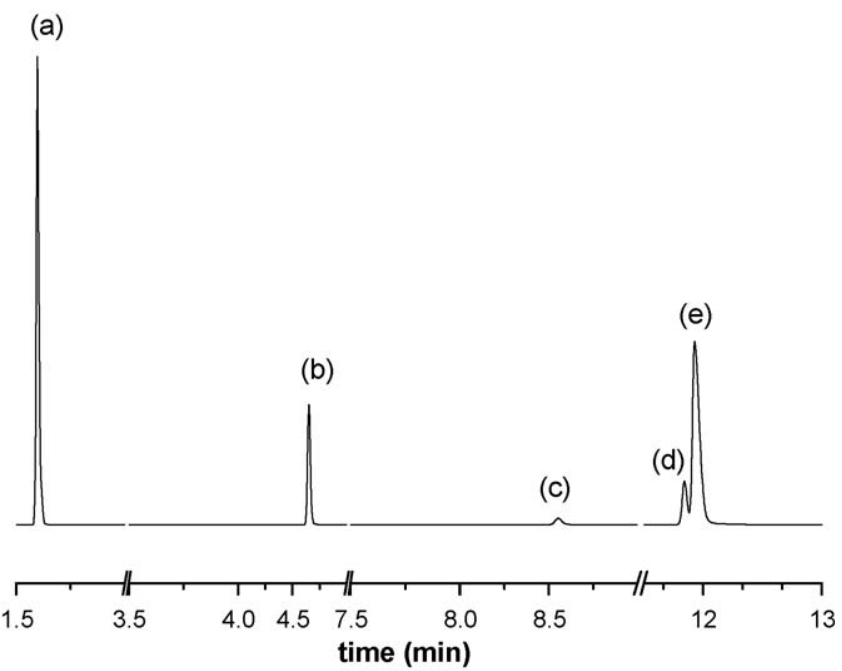

Fig. 1. Separation profile of the products in catalytic asymmetric addition of diethylzinc to benzaldehyde by gas chromatography (a) toluene (1.598 $\mathrm{min})$; (b) benzaldehyde (4.651 $\mathrm{min})$; (c) benzyl alcohol (8.554 min); (d) (R)-1-phenyl-1propanol (11.841 min); (e) (S)-1-phenyl-1-propanol (11.929 min).

EDX spectra. The results are shown in the supporting material (Table S1). For the mono-functionalized materials prepared by one-pot coprecipitation, the $\mathrm{Cl} / \mathrm{Si}$ ratio was proportional to the amount of CPTMS added in the synthesis gel, and the I.E. values were within 80-95\%. The I.E. values observed for the dualfunctionalized materials, however, exceeded $100 \%$, suggesting that TEOS did not hydrolyze completely. In contrast, for the material prepared by grafting method (30Cl-SBA-15-g), the loading of chloropropyl groups was relatively low, with an I.E. value of only $24 \%$. Moreover, the grafted sample has relatively low surface area and pore volume in comparison to the materials prepared by coprecipitation, inferring that the mesopores of the grafted sample may be blocked, to some extent, by the chloropropyl groups aggregated around the pore mouths.

\subsection{Proline-anchored SBA-15}

Proline derivatives were anchored onto functionalized SBA-15 through the nucleophilic substitution of chloropropyl groups with

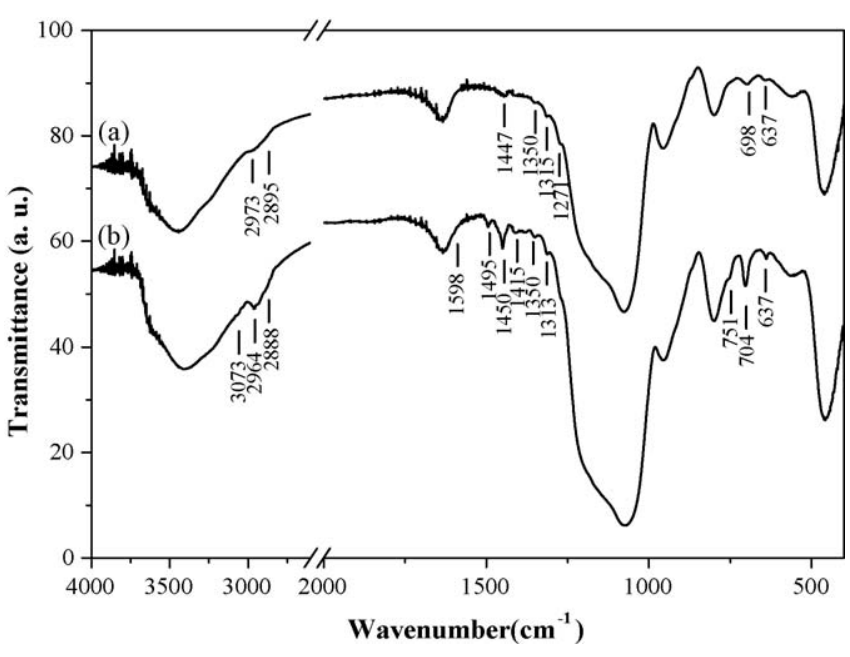

Fig. 3. IR spectra of (a) chloropropyl-functionalized SBA-15 and (b) prolineanchored SBA-15 materials.

(S)-(-)- $\alpha, \alpha$-diphenyl-2-pyrrolidinemethanol. The XRD patterns and $\mathrm{N}_{2}$ sorption isotherms of the resultant materials are shown in Fig. 2. All of them retain the ordered 2D-hexagonal $p 6 \mathrm{~mm}$ pore structure and type IV sorption isotherms with $\mathrm{H}_{1}$ hysteresis loops. The textural properties and the results of elemental analysis of proline-anchored SBA-15 materials are summarized in Table 1.

The IR spectra of chloropropyl-functionalized SBA-15 (10ClSBA-15-t) before and after proline-substitution are shown in Fig. 3. Both spectra exhibit common absorption branches at 468, 800, 1070 and $1220 \mathrm{~cm}^{-1}$ corresponding to the Si-O-Si bending and stretching vibrations of condensed silica framework along with the bending vibration of non-condensed silanol group at ca. $960 \mathrm{~cm}^{-1}$ [27] and the bending and stretching vibrations of hydroxyl groups and physically adsorbed water at 1650 and $3450 \mathrm{~cm}^{-1}$, respectively. Moreover, the peaks around 698, 1278-1433, and 2895$2973 \mathrm{~cm}^{-1}$ are attributed to the $\mathrm{C}-\mathrm{H}$ bending and stretching vibrations, which are notably enhanced with increasing chloropropyl loading. After proline-substitution, additional peaks due to the bending and stretching vibrations of aliphatic $\mathrm{C}-\mathrm{H}$ bonds appear at ca. 704, 1313-1450, and 2888-2964 $\mathrm{cm}^{-1}$; the out-ofplane bending and stretching vibrations of aromatic $\mathrm{C}-\mathrm{H}$ bonds appear at 751 and $3073 \mathrm{~cm}^{-1}$; and the stretching vibrations of
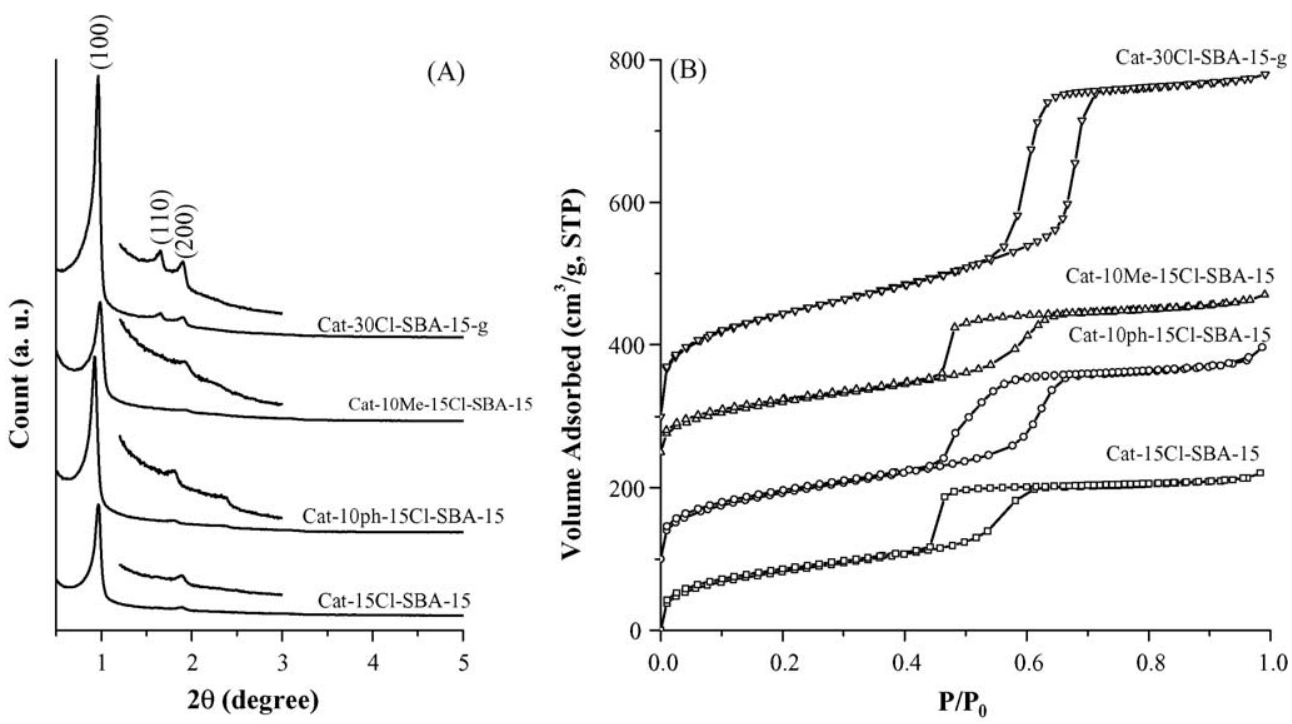

Fig. 2. (A) XRD patterns and (B) $\mathrm{N}_{2}$ adsorption/desorption isotherms of proline-anchored SBA-15 materials. 
Table 1

Elemental analysis and textural properties of proline-anchored mesoporous SBA-15 materials.

\begin{tabular}{|c|c|c|c|c|c|c|c|}
\hline Sample & $a_{0}{ }^{\mathrm{a}}(\mathrm{nm})$ & $S_{\mathrm{BET}}\left(\mathrm{m}^{2} / \mathrm{g}\right)$ & $V_{\text {Total }}\left(\mathrm{cm}^{3} / \mathrm{g}\right)$ & $D_{\mathrm{p}}^{\mathrm{b}}(\mathrm{nm})$ & $W_{\mathrm{t}}^{\mathrm{c}}(\mathrm{nm})$ & $N$ content $(\mathrm{mmol} / \mathrm{g})$ & I.E. of proline $\mathrm{d}^{\mathrm{d}}(\%)$ \\
\hline cat-5Cl-SBA-15 & - & 410 & 0.47 & 3.7 & - & 0.24 & 39 \\
\hline $10 \mathrm{Cl}-\mathrm{SBA}-15^{\mathrm{e}}$ & 10.8 & 885 & 0.88 & 4.5 & 6.3 & 0 & - \\
\hline cat-10Cl-SBA-15 & 10.3 & 342 & 0.39 & 3.6 & 6.7 & 0.36 & 25 \\
\hline cat-15Cl-SBA-15 & - & 305 & 0.33 & 3.3 & - & 0.49 & 25 \\
\hline cat-20Cl-SBA-15 & - & 280 & 0.23 & 3.4 & - & 0.41 & 17 \\
\hline $10 \mathrm{Cl}-\mathrm{SBA}-15-\mathrm{t}^{\mathrm{e}}$ & 10.7 & 704 & 0.69 & 4.3 & 6.4 & 0 & - \\
\hline cat-10Cl-SBA-15-t & 10.4 & 378 & 0.41 & 3.5 & 6.9 & 0.35 & 24 \\
\hline cat-15Cl-SBA-15-t & 10.6 & 331 & 0.41 & 3.4 & 6.2 & 0.46 & 23 \\
\hline cat-10Ph-15Cl-SBA-15-t & 11.1 & 272 & 0.33 & 3.4 & 7.5 & 0.25 & 10 \\
\hline cat-10Me-15Cl-SBA-15-t & 10.3 & 347 & 0.43 & 3.4 & 6.9 & 0.28 & 12 \\
\hline $30 \mathrm{Cl}-\mathrm{SBA}-15-\mathrm{g}^{\mathrm{e}}$ & 10.3 & 511 & 0.67 & 5.3 & 5.2 & 0 & - \\
\hline cat-30Cl-SBA-15-g & 10.5 & 530 & 0.73 & 4.8 & 5.7 & 0.39 & 44 \\
\hline cat-30Cl-SiO $2-\mathrm{g}$ & - & 335 & 0.29 & 3.4 & - & 0.44 & - \\
\hline
\end{tabular}

a $a_{0}=2 d_{100} / \sqrt{ } 3$

b Derived from the BJH desorption pore size distribution.

c Wall thickness calculated by $a_{0}-D_{\mathrm{p}}$.

d Incorporation efficiency of proline derivatives.

e Materials before anchoring proline.

aromatic ring appear at 1450,1495 , and $1598 \mathrm{~cm}^{-1}$ [30-32]. Nonetheless, the absorption peak at $637 \mathrm{~cm}^{-1}$, which may be attributed to the bending vibration of $\mathrm{C}-\mathrm{Cl}$ bond [33], is retained after proline-substitution, indicating that the substitution reaction was not complete. These results are consistent with those obtained from elemental analysis. On the other hand, no characteristic peaks corresponding to stretching and bending vibrations of $\mathrm{N}-\mathrm{H}$ bond are found, implying that there are no physically adsorbed $(S)-(-)$ $\alpha, \alpha$-diphenyl-2-pyrrolidinemethanol molecules on the material [27]. These results confirm that the proline derivatives are indeed anchored onto SBA-15 material through covalent bonding.

Solid-state ${ }^{29} \mathrm{Si}$ MAS and ${ }^{13} \mathrm{C}$ CP MAS NMR spectra of the cat10Cl-SBA-15-t sample are shown in Fig. 4. The ${ }^{29} \mathrm{Si}$ MAS NMR spectrum (Fig. 4A) shows three distinct resonance peaks at -94.1 , -102.1 , and $-110.9 \mathrm{ppm}$, which may be attributed to the $\mathrm{Q}^{2}, \mathrm{Q}^{3}$, and $\mathrm{Q}^{4}$ silicon species $\left(\mathrm{Q}^{n}=\mathrm{Si}(\mathrm{OSi})_{n}(\mathrm{OH})_{4-n}, n=2-4\right)$, respectively. Two additional peaks at -59.7 and $-66.5 \mathrm{ppm}$, respectively, due to the $\mathrm{T}^{2}$ and $\mathrm{T}^{3}$ silicons $\left(\mathrm{T}^{m}=\mathrm{RSi}(\mathrm{OSi})_{m}(\mathrm{OH})_{3-m}, m=1-3\right)[27,28,34]$ are also observed. The appearance of the $\mathrm{T}^{m}$ peaks confirms that the chloropropyl moiety is readily incorporated onto the silica framework through co-condensation of the CPTMS and TEOS precursors. Accordingly, the relative integrated intensities of the $\mathrm{T}^{m}$ and $\mathrm{Q}^{n}$ signals $\left(\mathrm{T}^{m} /\left(\mathrm{T}^{m}+\mathrm{Q}^{n}\right)\right)$ of 0.103 may be deduced, in good agreement with the theoretically expected value $(0.10)$ derived based on the composition of CPTMS in the initial mixture.

The ${ }^{13} \mathrm{C}$ CP-MAS NMR spectrum reveals three intense peaks at 9.4, 26.1, and $45.5 \mathrm{ppm}$ corresponding to the $\mathrm{C}$ atoms on the $\mathrm{O}_{3} \mathrm{Si}-$ $\mathrm{CH}_{2}-\mathrm{CH}_{2}-\mathrm{CH}_{2}-\mathrm{Cl}$ or $\mathrm{O}_{3} \mathrm{Si}-\mathrm{CH}_{2}-\mathrm{CH}_{2}-\mathrm{CH}_{2}-\mathrm{N}$ tethers displayed in sequence from left to right $[27,28,35,36]$ (Fig. 4B). The appearances of aromatic carbons at 126.6 and 146.2 ppm [28] and C atoms on proline at 56.2, 63.7, and $76.5 \mathrm{ppm}$ are also evident. These results demonstrate again that proline derivatives are successfully anchored onto the SBA-15 through nucleophilic substitution reaction. Nevertheless, the presence of the peaks at 15.7 and 70.4 ppm [37] indicate that small amounts of P123 residues still remain in the sample.

VT-HP ${ }^{129}$ Xe NMR spectroscopy was used to probe the distribution of proline groups on the SBA-15 materials prepared by different methods. Fig. 5 shows the VT-HP ${ }^{129}$ Xe NMR spectra of pristine and proline-anchored chloropropyl-functionalized SBA-15 materials prepared by one-pot co-condensation (cat-10Cl-SBA-15) and that prepared by grafting (cat-30Cl-SBA-15-g) method with similar CPTMS/(TEOS + CPTMS) molar ratios (0.10) together with the cat-10Cl-SBA-15-t sample post-treated with toluene. All spectra throughout the entire temperature range (180-298 K) reveal a broad, symmetric resonance line of adsorbed ${ }^{129} \mathrm{Xe}$ whose
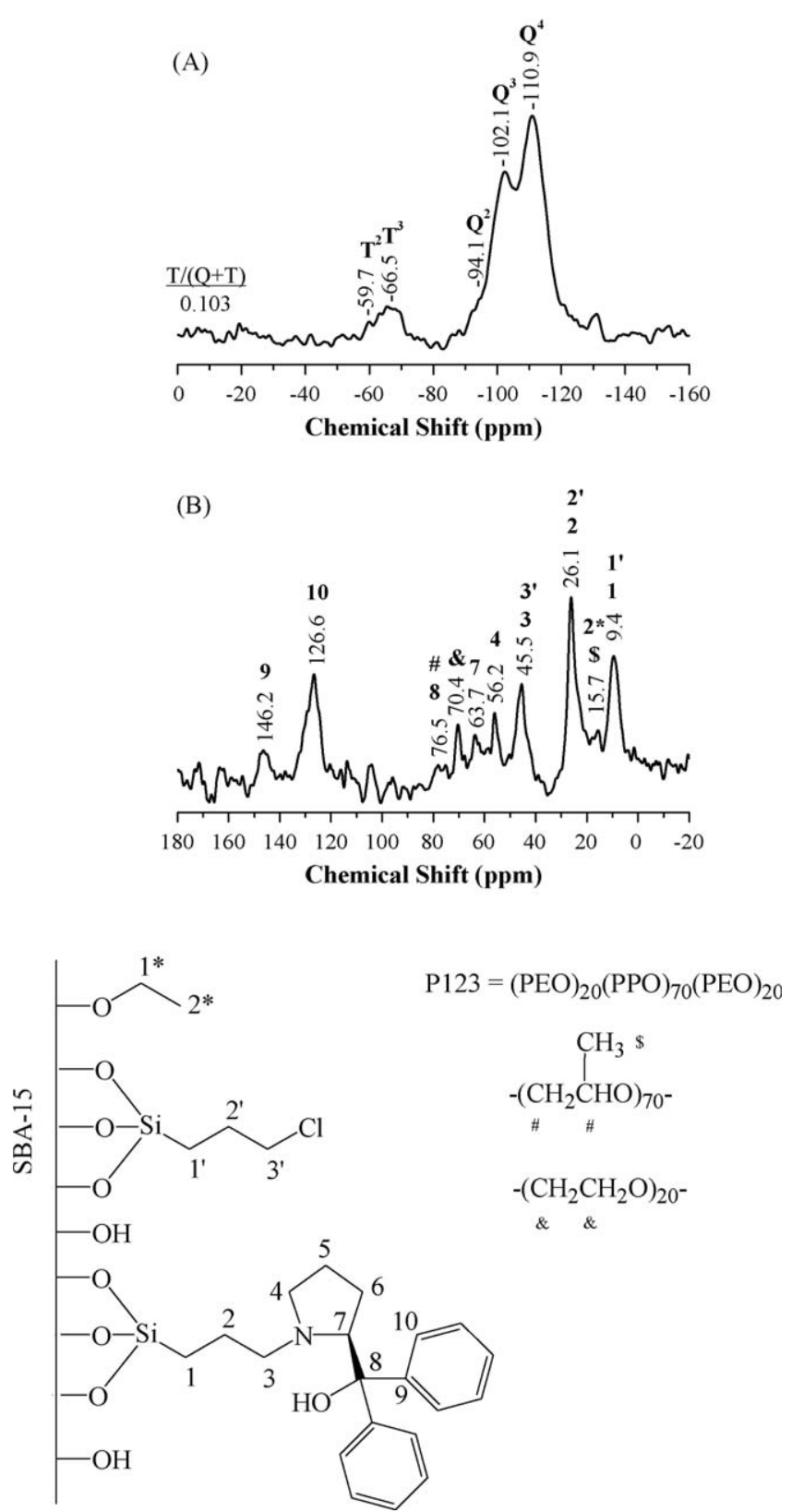

Fig. 4. (A) Solid-state ${ }^{29} \mathrm{Si}$ MAS and (B) ${ }^{13} \mathrm{C}$ CP-MAS NMR spectra of the cat- $10 \mathrm{Cl}-$ SBA-15-t material. 

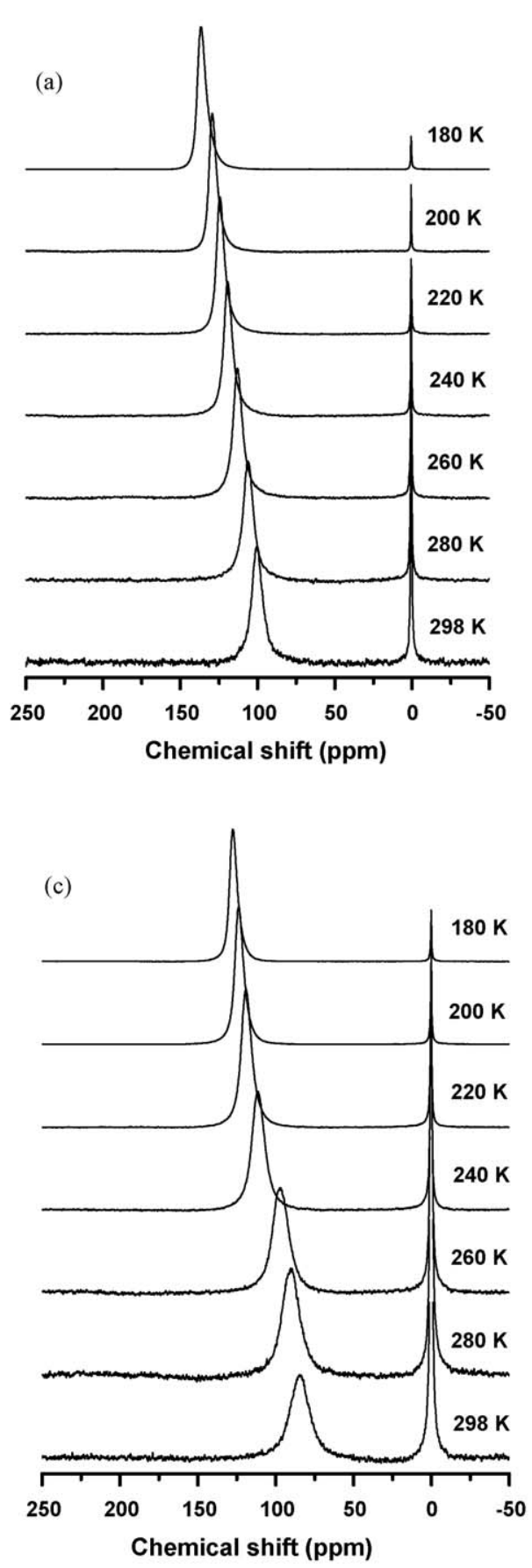
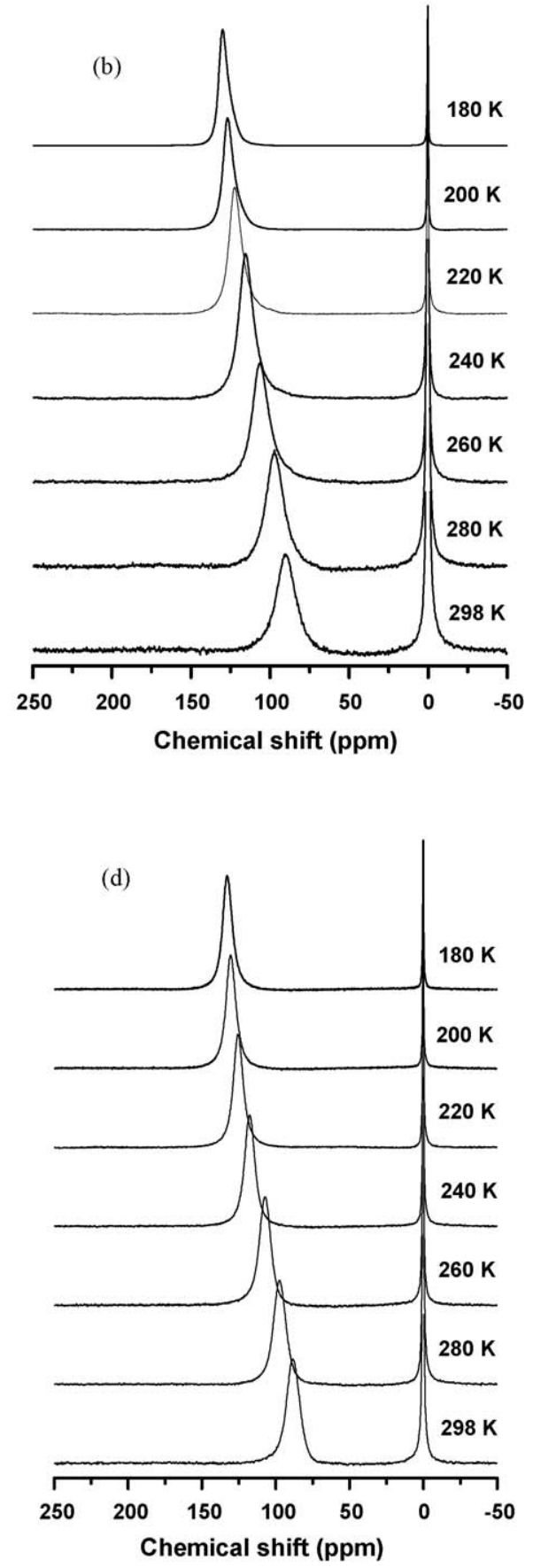

Fig. 5. VT-HP ${ }^{129}$ Xe NMR spectra of (a) 10Cl-SBA-15, (b) cat-10Cl-SBA-15, (c) cat-10Cl-SBA-15-t, and (d) cat-30Cl-SBA-15-g.

chemical shifts (80-155 ppm) are temperature dependent and a sharp, temperature-independent peak at ca. $0 \mathrm{ppm}$, which arises from gaseous ${ }^{129} \mathrm{Xe}$ in the inter-particle voids [38,39].

\subsection{Asymmetric addition of diethylzinc to benzaldehyde over proline- anchored SBA-15}

\subsubsection{Effect of reaction period}

The proline-anchored SBA-15 materials were applied as chiral selective catalysts for asymmetric addition of diethylzinc to benzaldehyde, leading to the formation of $(S)$-1-phenyl-propanol. Fig. 6 displays the conversion of benzaldehyde and product yields as a function of reaction period at $0{ }^{\circ} \mathrm{C}$ over the cat-15Cl-SBA-15- $t$ catalyst. The conversion of benzaldehyde decreases while the selectivity of main product (1-phenyl-1-propanol) increases almost linearly during the first reaction period of $5 \mathrm{~h}$. Then, the conversion slows down and the product yield reaches a plateau at a period of ca. $12 \mathrm{~h}$. In comparison, the yield of the minor 1-phenyl1-methanol by-product increases during the initial reaction period but slows down after $1.5 \mathrm{~h}$. For comparisons of the catalytic activities of various proline-anchored silica materials, only those results obtained before the reaction reaches its maximum are intended to be used. Hereafter, we have chosen to compare the results obtained from various catalysts at a reaction period of $8.5 \mathrm{~h}$.

\subsubsection{Effect of silica supports}

The conversion of benzaldehyde, selectivity of 1-phenyl-1propanol and enantiomeric excess of (S)-1-phenyl-1 propanol over 


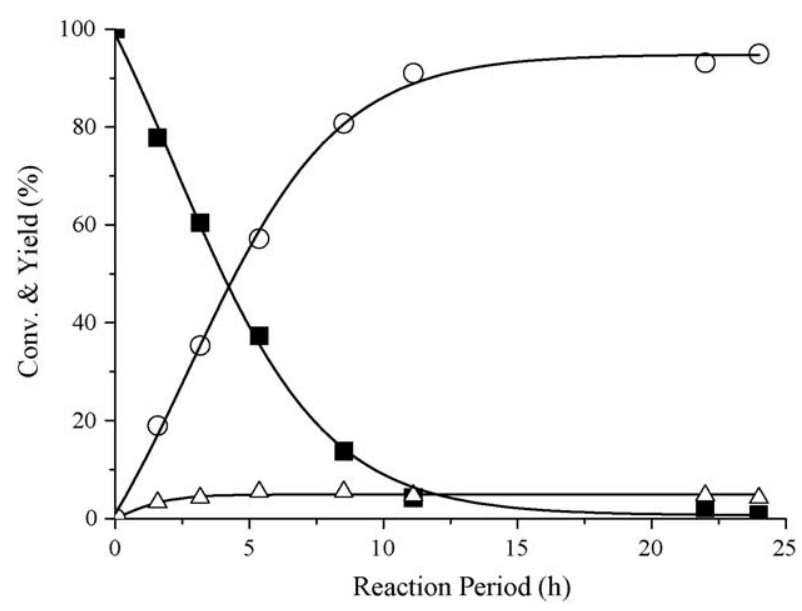

Fig. 6. The conversion of benzaldehyde $(\boldsymbol{\square})$ and yields of 1-phenyl-1-propanol ( $\bigcirc)$ and 1-phenyl-methanol $(\triangle)$ products as a function of reaction period obtained by using the cat-15Cl-SBA-15-t as a chiral selective catalyst.

various proline-anchored silica materials after reaction at $0{ }^{\circ} \mathrm{C}$ for $8.5 \mathrm{~h}$ are listed in Table 2 . In the absence of a catalyst (entry 1 ), $24 \%$ conversion of benzaldehyde and 60\% selectivity of 1-phenyl-1propanol were obtained. Over pure siliceous SBA-15 (entry 2), the conversion of benzaldehyde and selectivity of 1-phenyl-1-propanol increase to $60 \%$ and $80 \%$, respectively. However, no chiral selectivity was observed in either case. This implies that the addition reaction can proceed by just mixing benzaldehyde and diethylzinc, and that the reaction rate may be greatly enhanced when a solid substrate with large surface area (e.g., SBA-15) is present. Nevertheless, a chiral selective catalyst is inevitable for the formation of chiral selective products.

The asymmetric addition of benzaldehyde was achieved when proline-anchored silica materials were used as the catalysts (entries 3-8 of Table 2). However, the conversion of benzaldehyde and e.e. selectivity of (S)-1-phenyl-1-propanol seem to vary over a wide range. The catalytic activities of proline-anchored SBA-15 materials with different loadings of proline derivatives are shown in entries 3 to 6; here the pristine chloropropyl-SBA-15 materials for anchoring proline were prepared by the co-condensation method. The conversion of benzaldehyde and e.e. value are found to increase dramatically with increasing proline loading. The highest benzaldehyde conversion of $94 \%$ and the e.e. value of $51 \%$ were obtained over the cat-15Cl-SBA-15 catalyst, which has a proline loading of $0.49 \mathrm{mmol} / \mathrm{g}$. However, it is also noticed that the catalytic activity decreases markedly over the cat-20Cl-SBA-15 sample, which has the highest chloropropyl content but slightly lower proline loading. The poor catalytic activity and enantiometric excess over the cat-20Cl-SBA-15 material are attributed to the lower surface area and porosity. These results indicate that the catalytic activity and enantiometric excess depend mainly on the loading of proline derivatives, however, high surface area and porosity of the support are also important.

\subsubsection{Effect of different preparation methods of functionalized silica support}

Entry 7 of Table 2 shows that cat-30Cl-SBA-15-g, which has proline derivative on chloropropyl-functionalized SBA-15 prepared by post-grafting method, gives $92 \%$ conversion of benzaldehyde and $92 \%$ selectivity of 1-phenyl-1-propanol. However, it gives only $25 \%$ e.e. selectivity. Although this catalyst has a similar loading of proline derivatives, it possesses much higher surface area and pore volume than the cat-10Cl-SBA-15 and the cat-20Cl-SBA-15 catalysts. Such results are indicative that not all of the proline sites are effective as chiral selective catalysts. The proline groups in cat-30Cl-SBA-15-g are probably aggregated near the mouths of mesopores, as discussed in Section 4. On the other hand, the proline-anchored amorphous silica (entry 8 , cat-30Cl-SiO ${ }_{2}-$ g) gave a much higher e.e. of $51 \%$ but lower conversion of benzaldehyde and selectivity of 1-phenyl-1propanol (46\% and 78\%, respectively). This implies that the distributions of proline derivatives on cat-30Cl-SBA-15-g and cat$30 \mathrm{Cl}-\mathrm{SiO}_{2}$-g materials are probably different.

Among the proline-anchored silica catalysts, mesoporous cat15Cl-SBA-15 gives the highest conversion of benzaldehyde, selectivity of 1-phenyl-1-propanol, and e.e. selectivity of (S)-1phenyl-1 propanol.

\subsubsection{Effect of post-treatment with toluene}

The textural properties and catalytic activities of cat-15-SBA-15 after removing P123 residue with toluene prior to anchoring proline derivative are shown in Table 3 . In comparison with the material without toluene treatment, the surface area and pore volume of the post-treated material are higher due to more thorough removal of residual P123 template. During the asymmetric addition of benzaldehyde with diethylzinc, although the benzaldehyde conversion decreases from $94 \%$ to $86 \%$, the e.e. value increases from $51 \%$ to $66 \%$. As shown in entry 3 of Table 3 , the catalytic activity, selectivity of 1-phenyl-1-propanol, and e.e. value were enhanced to $98 \%, 97 \%$ and $69 \%$, respectively, when double the amount of the catalyst was used. In contrast, if the reaction temperature is increased from $0{ }^{\circ} \mathrm{C}$ to room temperature, the benzaldehyde conversion could be further increased to $99 \%$ but the e.e. value dropped to $31 \%$ (entry 4 ).

\subsubsection{Effect of co-functionalizing with hydrophobic groups}

In addition to the chloropropyl groups on SBA-15 which were used for anchoring the proline derivative, methyl or phenyl groups were also introduced on SBA-15 by one-pot co-condensation method in order to increase surface hydrophobicity. The physicochemical properties and catalytic activities of these dual-functionalized materials are shown in entries 6 and 7 of Table 3. In

Table 2

The asymmetric addition of diethylzinc to benzaldehyde over various proline-anchored silica materials. ${ }^{\mathrm{a}}$.

\begin{tabular}{|c|c|c|c|c|c|c|c|}
\hline Entry & Catalysts & $S_{\text {BET }}\left(\mathrm{m}^{2} / \mathrm{g}\right)$ & $V_{\text {Total }}\left(\mathrm{cm}^{3} / \mathrm{g}\right)$ & $N$ content $(\mathrm{mmol} / \mathrm{g})$ & Conversion (\%) & Selectivity $^{\mathrm{b}}(\%)$ & e.e. (\%) \\
\hline 1 & Null & - & - & - & 24 & 60 & 0 \\
\hline 2 & Pure SBA-15 & 779 & 1.0 & - & 60 & 80 & 0 \\
\hline 3 & cat-5Cl-SBA-15 & 410 & 0.47 & 0.24 & 33 & 88 & 18 \\
\hline 4 & cat-10Cl-SBA-15 & 342 & 0.39 & 0.36 & 81 & 95 & 37 \\
\hline 5 & cat-15Cl-SBA-15 & 305 & 0.33 & 0.49 & 94 & 96 & 51 \\
\hline 6 & cat-20Cl-SBA-15 & 280 & 0.23 & 0.41 & 23 & 74 & 23 \\
\hline 7 & cat-30Cl-SBA-15-g & 530 & 0.73 & 0.39 & 92 & 92 & 25 \\
\hline 8 & cat-30Cl-SiO ${ }_{2}-g^{\mathrm{d}}$ & 335 & 0.29 & 0.44 & 46 & 78 & 51 \\
\hline
\end{tabular}

a Reaction condition: $25 \mathrm{mg}$ catalyst, $0{ }^{\circ} \mathrm{C}$ for $8.5 \mathrm{~h}$; diethylzinc/benzaldehyde $=2.4$.

b Selectivity of 1-phenyl-1-propanol.

c Chloropropyl-SBA-15 prepared by post-grafting method.

d Chloropropyl-silica gel prepared by post-grafting method. 
Table 3

The asymmetric addition of diethylzinc to benzaldehyde over proline-anchored SBA-15 post-treated with toluene. ${ }^{\text {a }}$.

\begin{tabular}{|c|c|c|c|c|c|c|c|c|c|}
\hline Entry & Catalysts & $S_{\mathrm{BET}}\left(\mathrm{m}^{2} / \mathrm{g}\right)$ & $V_{\text {Total }}\left(\mathrm{cm}^{3} / \mathrm{g}\right)$ & $N$ content $(\mathrm{mmol} / \mathrm{g})$ & Method $^{\mathrm{b}}$ & Conversion (\%) & TON $\left(\times 10^{-4} \mathrm{~s}^{-1}\right)$ & Selectivity ${ }^{\mathrm{c}}(\%)$ & e.e. $(\%)$ \\
\hline 1 & cat-15Cl-SBA-15 & 305 & 0.33 & 0.49 & A & 94 & 12 & 96 & 51 \\
\hline 2 & cat-15Cl-SBA-15-t & 331 & 0.41 & 0.46 & A & 86 & 12 & 98 & 66 \\
\hline 3 & cat-15Cl-SBA-15-t ${ }^{\mathrm{d}}$ & 331 & 0.41 & 0.46 & A & 98 & 13 & 97 & 69 \\
\hline 4 & cat-15Cl-SBA-15- $\mathrm{t}^{\mathrm{e}}$ & 331 & 0.41 & 0.46 & A & 99 & 13 & 92 & 31 \\
\hline 5 & cat-15Cl-SBA-15-t & 331 & 0.41 & 0.46 & B & 83 & 11 & 97 & 75 \\
\hline 6 & cat-10Me-15Cl-SBA-15-t & 347 & 0.43 & 0.28 & A & 97 & 22 & 97 & 65 \\
\hline 7 & cat-10Ph-15Cl-SBA-15-t & 272 & 0.33 & 0.25 & A & 73 & 18 & 96 & 55 \\
\hline
\end{tabular}

a Reaction condition: $25 \mathrm{mg}$ catalyst, $0{ }^{\circ} \mathrm{C}$ for $8.5 \mathrm{~h}$.

b Method A: diethylzinc/benzaldehyde $=2.4$; method B: diethylzinc/benzaldehyde $/ n$-butyl lithium $=2.4 / 1.0 / 5.1 \times 10^{-3}$.

c Selectivity of 1-phenyl-1-propanol.

d Double amount ( $50 \mathrm{mg}$ ) of the catalyst was used.

e Reaction temperature at $25^{\circ} \mathrm{C}$.

comparison with that of mono-functionalized cat-15Cl-SBA-15-t, the proline loadings on these dual-functionalized materials are much lower. This may be attributed to the greater difficulty for proline derivative to access chloropropyl groups during substitution reaction. However, the benzaldehyde conversion, 1-phenyl-1propanol selectivity and e.e. value reached very high values of $97 \%$, $97 \%$ and $65 \%$, respectively, over methyl co-functionalized cat10Me-15Cl-SBA-15-t. On the other hand, phenyl co-functionalized cat-10Ph-15Cl-SBA-15-t gave relatively lower catalytic activity and e.e. selectivity.

\subsubsection{Effect of n-butyl lithium addition on catalytic activity}

As shown in entry 5 of Table 3 , the conversion of benzaldehyde is slightly decreased but the enantiometric excess of (S)-1-phenyl1-propanol is significantly increased over cat-15Cl-SBA-15-t upon addition of a small amount of $n$-butyl lithium.

\subsubsection{Reuse of the catalyst}

In order to examine the possibility of reusing the catalyst, the spent catalyst was separated from the liquid products by filtration and treated with $0.2 \mathrm{M} \mathrm{HCl}$ to dissolve zinc hydroxide precipitate. After being washed with deionized water and ethanol, the solid was dried at $100{ }^{\circ} \mathrm{C}$. Before the catalyst was reused, the solid was neutralized with $0.2 \mathrm{M} \mathrm{TMAOH} / \mathrm{MeOH}$ and dried again. Table 4 shows the catalytic activities of cat-15Cl-SBA-15-t after regeneration and reuse. The benzaldehyde conversion decreases slightly from $86 \%$ to $84 \%$ after the first regeneration, and to $76 \%$ after the second regeneration. The decrease in conversion is attributed to the loss of the solid catalyst during regeneration procedure. On the other hand, the selectivity of 1-phenyl-1-propanol and enantiometric excess are well retained at $96-98 \%$ and $72-74 \%$, respectively. These results indicate that the proline-anchored SBA-15 materials are stable during the reaction and can be easily regenerated and reused after filtration, washing with acid, and neutralization.

\section{Discussion}

\subsection{Distribution of functional groups}

After anchoring the proline derivative, the surface area and pore volume of the functionalized SBA-15 samples prepared by one-pot method decrease markedly, while those of the sample prepared by

Table 4

Catalytic activities of the cat-15Cl-SBA-15-t catalyst before and after repeated regeneration.

\begin{tabular}{llll}
\hline Number of regeneration & Conversion (\%) & Selectivity (\%) & e.e. (\%) \\
\hline 0 & 86 & 98 & 66 \\
1 & 84 & 97 & 65 \\
2 & 76 & 96 & 64 \\
\hline
\end{tabular}

grafting method are hardly changed (Table 1 ). Moreover, the unit cell parameters shrank slightly for the functionalized SBA-15 samples prepared by one-pot method but remain practically unchanged for the post-grafted samples. These results imply that the materials prepared by one-pot method contain significant amount of silanol groups on the framework [26-28,34], which are subjected to further condensation during the nulceophilic substitution reaction.

The nitrogen content in the functionalized SBA-15 prepared by one-pot method first increases with the loading of chloropropyl groups to reach a maximum at $0.49 \mathrm{mmol} / \mathrm{g}$ for the cat-15Cl-SBA15 sample, then, decreases slightly to $0.41 \mathrm{mmol} / \mathrm{g}$ as the chloropropyl loading is further increased, due to the decrease in surface area and pore volume on the more perturbed functionalized material (cat-20Cl-SBA-15). The incorporation efficiencies (I.E.) of proline derivatives, which span over the range of $17-39 \%$, are found to decrease with increasing chloropropyl loading. These results indicate that less than $40 \%$ of the chloropropyl groups are successfully substituted by the proline derivatives. On the other hand, higher surface area and pore volume of the proline-anchored SBA-15 materials are obtained when the functionalized SBA-15 is post-treated with toluene. However, the pore diameter, $\mathrm{N}$ content, and I.E. value of proline are similar to those without the posttreatment. These phenomena can be readily seen by comparing the results observed for the cat-15Cl-SBA-15 and the cat-15Cl-SBA-15t samples.

It is noted that the proline contents observed for the functionalized SBA-15 and amorphous silica prepared by postgrafting are in general higher than those prepared by the one-pot method. For example, the I.E. value of proline reaches the highest value of $44 \%$ on the cat-30Cl-SBA-15-g sample. These results suggest that the grafted chloropropyl groups are probably aggregated near the pore mouths of SBA-15 and amorphous silica, and they are readily substituted by the proline derivatives.

If one compares the proline-anchored dual-functionalized SBA15 materials (i.e., cat-10Ph-15Cl-SBA-15-t and cat-10Me-15ClSBA-15-t) with the mono-functionalized material (cat-15Cl-SBA$15-t$ ), the nitrogen contents observed for the former two samples are only ca. $60 \%$ of the latter, suggesting that the hydrophobic methyl and phenyl groups are probably unfavorable for the access of proline to the chloropropyl sites during the substitution reaction. In addition, the surface area, pore volume, and nitrogen content of the methyl co-functionalized sample are all higher than those of the phenyl co-functionalized sample, inferring that the larger phenyl groups may, to some extent, hinder the diffusion of bulky (S)-(-)- $\alpha, \alpha$-diphenyl-2-pyrrolidinemethanol molecules into the deep interior of the mesopore channels. As a result, a relatively larger amount of the proline derivatives may aggregate around the pore mouths.

The observation of a single resonance line of adsorbed ${ }^{129} \mathrm{Xe}$ reflects that an exchange of xenon among different adsorption sites 


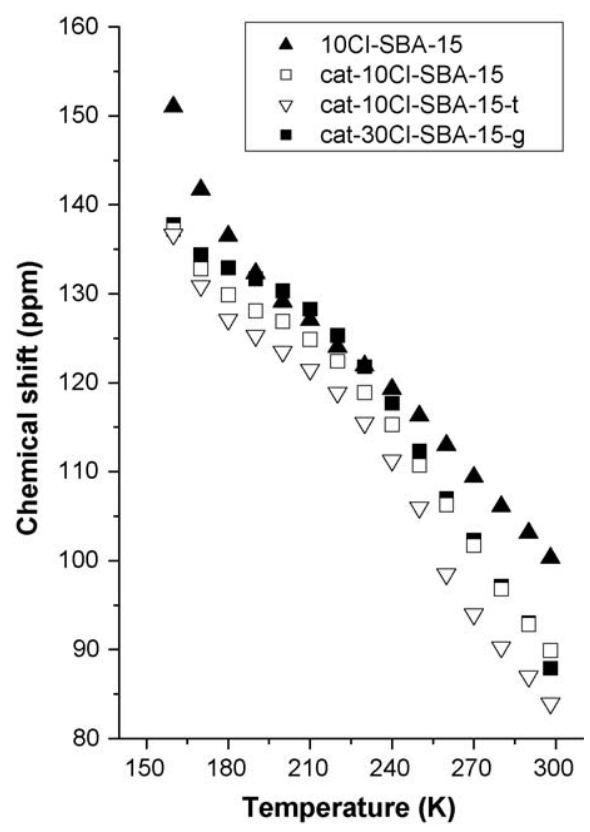

Fig. 7. Variations of VT-HP ${ }^{129} \mathrm{Xe}$ NMR chemical shifts with temperature for xenon adsorbed on 10Cl-SBA-15, cat-10Cl-SBA-15, cat-10Cl-SBA-15-t, and cat-30Cl-SBA15 -g materials.

should be anticipated. Thus, an averaged local environment of the xenon atoms in the experimental NMR time scale was probed. SBA15 is a well-known mesoporous silica which may contain micorpores on the walls. A fast exchange of xenon among micropores and mesopores in SBA-15 was reported to give rise to a symmetric peak in the temperature range of 160-298 K [39]. The variations of $\mathrm{HP}{ }^{129} \mathrm{Xe}$ NMR chemical shifts with temperature observed for different samples are shown in Fig. 7. As the temperature decreases, the ${ }^{129} \mathrm{Xe}$ chemical shift is increased due to an increasing amount of xenon being adsorbed in the pore channels. As the temperature further decreases to a critical point $\left(T_{\mathrm{cc}} \sim 170-190 \mathrm{~K}\right)$, an abrupt increase in ${ }^{129} \mathrm{Xe}$ chemical shift is observed due to condensation of xenon. It is noted that the temperature dependence of the chemical shift for $10 \mathrm{Cl}$-SBA-15 is rather different from those for other samples, revealing a higher (ca. $190 \mathrm{~K}$ ) threshold temperature of condensation $\left(T_{c c}\right)$, which should be pore size dependent. Presumably, sample with a smaller pore size should be more prone to capillary condensation at a higher temperature, as previously observed for pure siliceous SBA15 [35]. In view of the fact that the 10Cl-SBA-15 sample has an average mesopore size $\left(D_{\mathrm{p}}=4.5 \mathrm{~nm}\right)$ larger than those of the cat10Cl-SBA-15 $\left(D_{\mathrm{p}}=3.6 \mathrm{~nm}\right)$ and cat-10Cl-SBA-15-t $\left(D_{\mathrm{p}}=3.5 \mathrm{~nm}\right)$ samples (which both show $T_{\mathrm{cc}} \sim 170 \mathrm{~K}$ ), one can infer that the 10Cl-SBA-15 sample should contain more micropores. In contrast, substantial blocking of micropores may occur for the prolineanchored materials, making them inaccessible to xenon. These results are evidenced by the data of microporous surface area and volume derived from the $t$-plots of $\mathrm{N}_{2}$ sorption isotherms (Table 5). A significant decrease of the microporous surface area and volume was observed after 10Cl-SBA-15 was anchored by proline derivative.

The temperature dependence of $\mathrm{HP}{ }^{129} \mathrm{Xe}$ chemical shift can be approximated by an equation derived based on a fast-exchange model in the Henry Law region [38]:

$\delta=\frac{\delta_{\mathrm{s}}}{1+V / S K_{0} R T^{1 / 2} \exp (\Delta H / R T)}$

where $V$ and $S$ represent the free volume and specific surface area of the mesoporous materials, respectively. $K_{0}$ is the pre-exponent of Henry's constant, $R$ is the universal gas constant, $\Delta H$ is the heat of adsorption, and $\delta_{\mathrm{s}}$ is the characteristic chemical shift reflecting the xenon-surface interaction. Accordingly, the parameters obtained for various samples by least-square fittings of ${ }^{129} \mathrm{Xe}$ chemical shift variations with temperature to Eq. (3) are depicted in Table 5. Since the chemical shift contribution arising from Xe-Xe interactions becomes more significant at low temperature, only experimental data obtained at $T>T_{\mathrm{cc}}$ were included during the fittings. The $\Delta H$ values obtained for various samples are all in the range of $10-14 \mathrm{~kJ} / \mathrm{mol}$, implying that the Xe atoms are physically adsorbed.

The $\delta_{\mathrm{s}}$ value, which reflects interactions between xenon and pore surfaces, has been shown to have strong correlations with the coverage, chain length, and functional group of organic moieties on the surfaces $[29,40]$ and can be regarded as the sum of two contributions, namely $\delta_{\text {wall }}$ and $\delta_{\text {moiety }}$ arising from Xe-silica wall and Xe-moiety interactions, respectively. Presumably, when more organic moieties are introduced onto the surface, the $\delta_{\mathrm{s}}$ value would be increased due to Xe-moiety interactions. Here, however, the $\delta_{\mathrm{s}}$ values observed for cat-10Cl-SBA-15 and cat-10Cl-SBA-15-t samples are smaller than that for 10Cl-SBA-15. This observation, together with the larger $V / S K_{0}$ values and the lower $T_{\mathrm{cc}}$ observed for the proline-anchored materials compared to the 10Cl-SBA-15 sample, again, implies that anchoring of proline led to blockage of microporosity.

The fact that the $\delta_{\mathrm{s}}$ and $V / S K_{0}$ values found for cat-30Cl-SBA-15$\mathrm{g}$ are larger than those of cat-10Cl-SBA-15-t should reflect variations in the porous structure and proline coverage. In this case, the increase in $V / S K_{0}$ value for cat-30Cl-SBA-15-g can be attributed to the increase in the $V_{\text {Total }} / S_{\mathrm{BET}}$ ratio, suggesting that it has a larger pore size than cat-10Cl-SBA-15-t. Indeed, the BJH pore diameter of cat-30Cl-SBA-15-g determined from nitrogen sorption isotherm decreases by only ca. $0.5 \mathrm{~nm}$ compared to 30Cl-SBA-15-g, suggesting that aggregation of the proline moieties near the pore mouths is more plausible to occur. On the other hand, the larger $\delta_{\mathrm{s}}$ value observed for cat-30Cl-SBA-15-g than for cat-10Cl-SBA-15-t reflects the fact that Xe atoms can gain access to silica wall and organic moieties more easily. Huang et al. deduced that the bulky proline moieties should either stand up on the internal surfaces or

Table 5

Textural properties derived from $\mathrm{N}_{2}$ sorption isotherms and parameters in Eq. (3) of various functionalized SBA-15 materials.

\begin{tabular}{|c|c|c|c|c|c|c|c|}
\hline Sample $^{a}$ & $V_{\text {Total }} / S_{\text {BET }}\left(10^{-9} \mathrm{~m}\right)$ & $S_{\text {Micro }}{ }^{b}\left(\mathrm{~m}^{2} / g\right)$ & $V_{\text {Micro }}{ }^{\mathrm{b}}\left(\mathrm{cm}^{3} / \mathrm{g}\right)$ & $D_{\mathrm{p}}{ }^{\mathrm{c}}(\mathrm{nm})$ & $\delta_{\mathrm{s}}(\mathrm{ppm})$ & $V / S K_{0}\left(10^{-20}\right.$ Torr $\left.\mathrm{m}^{3}\right)$ & $\Delta H(\mathrm{~kJ} / \mathrm{mol})$ \\
\hline 10Cl-SBA-15 & 0.99 & 123 & 0.045 & 4.5 & $135.9 \pm 0.7$ & $0.0045 \pm 0.0006$ & $10.6 \pm 0.3$ \\
\hline cat-10Cl-SBA-15 & 1.14 & 26 & 0.004 & 3.6 & $132.9 \pm 0.9$ & $0.016 \pm 0.003$ & $12.9 \pm 0.5$ \\
\hline cat-10Cl-SBA-15-t ${ }^{\mathrm{d}}$ & 1.08 & 19 & 0.002 & 3.5 & $130.7 \pm 1.4$ & $0.019 \pm 0.005$ & $12.9 \pm 0.7$ \\
\hline cat-30Cl-SBA-15-ge & 1.38 & 44 & 0.010 & 4.8 & $135.8 \pm 0.6$ & $0.026 \pm 0.004$ & $13.8 \pm 0.4$ \\
\hline
\end{tabular}

\footnotetext{
a All proline-anchored samples were prepared after TMAOH neutralization treatment.

b Micrporous surface area and volume determined by $t$-plot method.

c Derived from the BJH desorption pore size distribution.

d Post-treated with toluene before anchoring proline.

e Chloropropyl-SBA-15 precursor was prepared by post-grafting method.
} 


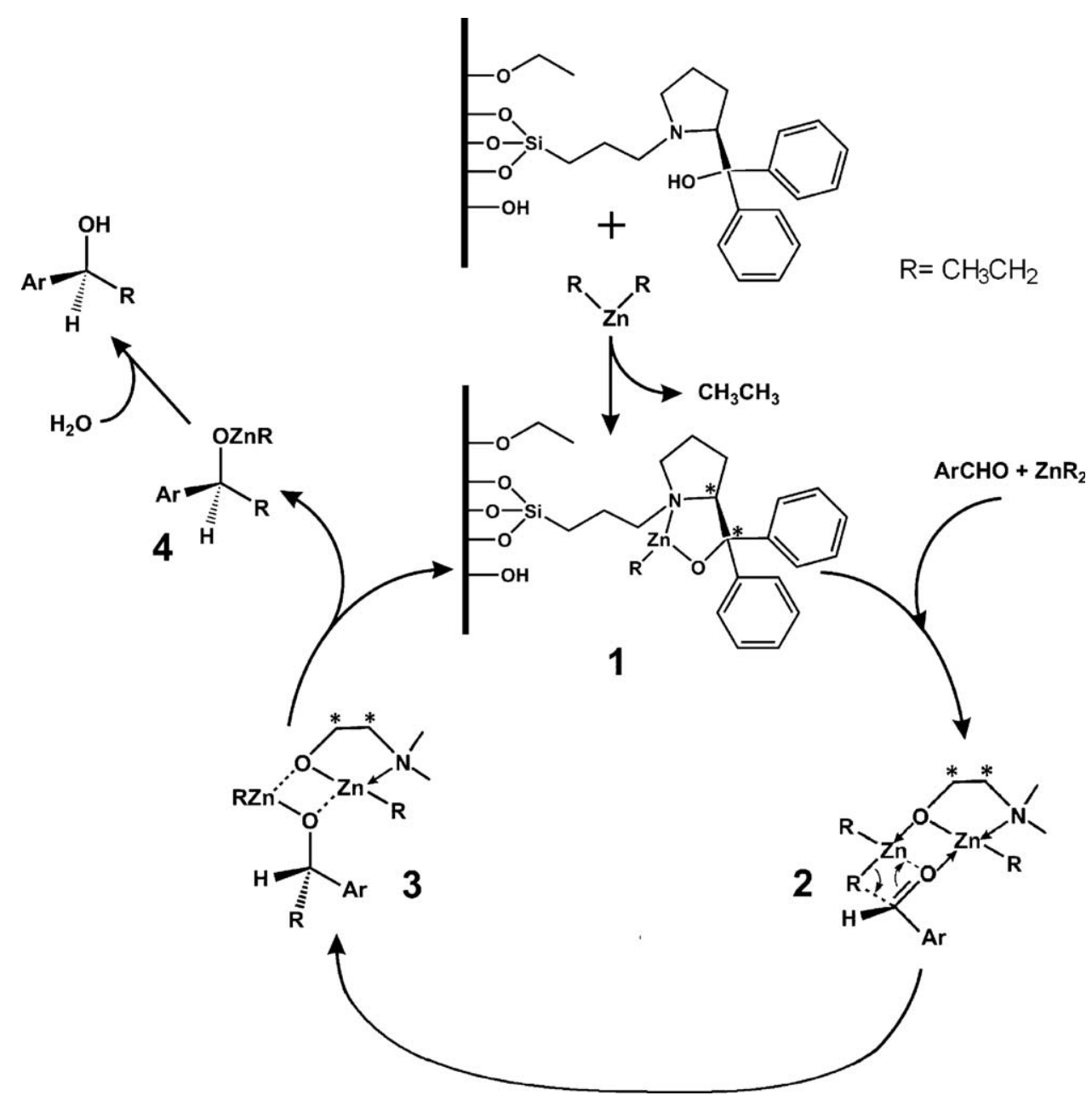

Scheme 3. Possible mechanism of asymmetric addition of diethylzinc to benzaldehyde over proline-anchored silica surfaces.

form aggregated clusters near the pore mouths in the case of cat30Cl-SBA-15-g, in which Xe may be "dissolved" in the organic phase [29,39], whereas the proline moieties in cat-10Cl-SBA-15-t are probably laid down homogeneously on the internal pore surfaces.

\subsection{Catalytic activities}

The asymmetric addition of benzaldehyde was achieved when proline-anchored silica materials were used as the catalysts. The catalytic activity and enantiometric excess were found to depend mainly on the loading of proline derivatives; however, the high surface area and porosity of the support are also important. The reaction mechanism of asymmetric addition of benzaldehyde with diethylzinc has been studied by Noyori and Kitamura [41] using (-)-3-exo-(dimethylamino)isoborneol as a chiral selective catalyst. A similar mechanism is proposed over the proline-anchored silica surfaces, as shown in Scheme 3. After elimination of an ethane molecule from diethylzinc, the anchored chiral catalyst is believed to coordinate with zinc through amine and alcohol sites to form a five-member ring intermediate $\mathbf{1}$. This intermediate further reacts with benzaldehyde through zinc and another diethylzinc molecule through oxygen, and then the resulting chiral-selective transition species $\mathbf{2}$ facilitate the ethyl-migration from diethylzinc to aldehyde via a planar bicyclic transition state. The optical active (S)-1-phenyl-1-propanol is obtained by hydrolysis of 1-phenyl-1propanol zincate 4 in an acidic aqueous solution. On the other hand, 1-phenyl-1-methanol is obtained as a by-product through $\mathrm{H}$ migration from diethylzinc to aldehyde, as shown in Scheme 4.
According to Noyori and Kitamura [41], the backbone of the chiral auxiliary defines the chirality of the bridgehead atoms in the stereo-determining bicyclic intermediates $\mathbf{5}$ and $\mathbf{6}$, which in turn is transmitted to the aldehyde ligand by an alkyl transfer reaction. With the $\alpha S$ configurated amino alcohol ancillaries, the $S$ generating transition states $\mathbf{5}$ is favored over the diastereomeric $R$-transition states $\mathbf{6}$, which probably suffers significant nonbonded repulsion between $\mathrm{Ar}$ and the $\mathrm{Zn}_{\mathrm{A}}$-linked $\mathrm{R}$ group.
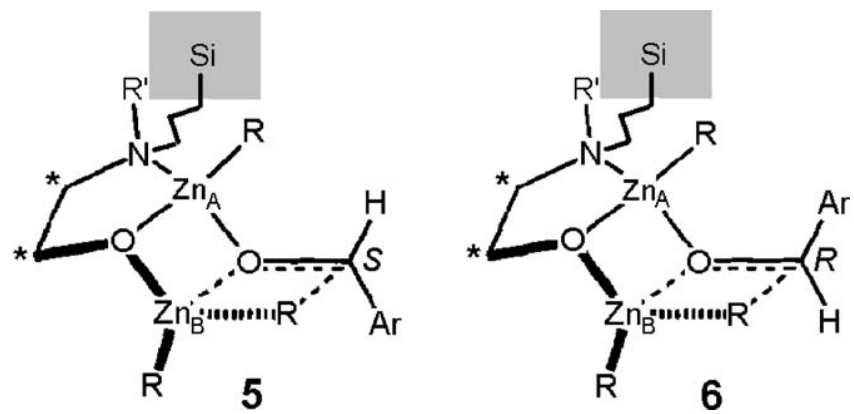

The higher catalytic activity and e.e. selectivity values of functionalized SBA-15 materials prepared by co-condensation

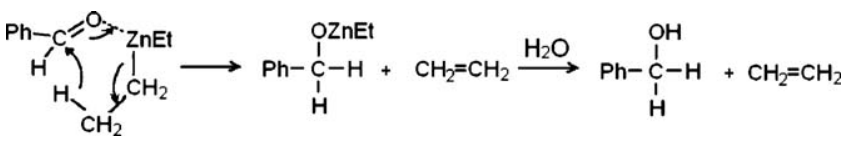

Scheme 4. Possible mechanism of the formation of 1-phenyl-1-methanol byproduct. 
method are attributed to the fact that the chloropropyl groups are more homogeneously distributed in the mesopores of SBA-15 material prepared by the co-condensation method than in that prepared by the post-grafting method. After nucleophilic substitution of chloropropyl groups with (S)-(-)- $\alpha, \alpha$-diphenyl-2pyrrolidinemethanol, the resultant proline derivatives are also better dispersed inside the pores of SBA-15, as evidenced by the results of nitrogen sorption isotherms and Xe NMR spectra. The homogeneous distribution of the functional groups avoids the interaction between nearby proline groups, and makes each site an effective catalyst in asymmetric addition.

The proline derivative samples of chloropropyl-functionalized SBA-15 and amorphous silica prepared by post-grafting method give very different activities in terms of benzaldehyde conversion, 1-phenyl-1-propanol selectivity, and e.e. selectivity. Since amorphous silica contains a broad range of pores, the chloropropyl groups are more likely to be grafted on the surfaces of larger pores rather than aggregated near the openings of the smaller pores. After nucelophilic substitution, the bulky (S)-(-)- $\alpha, \alpha$-diphenyl-2pyrrolidinemethanol compounds should be anchored on the open pores of cat-30Cl-SiO ${ }_{2}$-g and their distribution is probably more homogeneous and less crowded than that on cat-30Cl-SBA-15-g. Since it was reported that the enanotiometric excess of products could be increased by decreasing the steric hindrances of chiral selective catalysts [42], the cat-30Cl- $\mathrm{SiO}_{2}-\mathrm{g}$ catalyst that has a better dispersion of proline derivatives than cat-30Cl-SBA-15-g shows a higher selectivity to $(S)$-phenyl-1-propanol product.

The reason that the catalysts treated with toluene displayed lower activity but higher enantiometric excess is the higher efficiency of the better dispersed proline sites in the post-treated samples. As for the lower conversion observed, it is probably due to the more hydrophilic nature of the SBA-15 surfaces after the residual P123 has been extracted. The conversion can be easily improved by using a larger amount of the catalyst.

It has been shown that the catalytic activity and e.e. selectivity are enhanced by incorporation of additional methyl groups on the surface of chloropropyl-functionalized SBA-15. On the contrast, the incorporation of phenyl groups has negative effect. Between methyl and phenyl groups, the methyl group is smaller and more hydrophobic than the phenyl group. The surface area, pore volume, and pore diameter of the methyl co-functionalized SBA-15 material are better retained than those of the phenyl cofunctionalized sample. Moreover, the introduction of small methyl groups has less effect on the conformation and flexibility of the catalytic proline sites. Therefore, the benzaldehyde addition reaction proceeds faster over the methyl co-functionalized cat10Me-15Cl-SBA-15-t material with a high enantiomeric excess than over the mono-functionalized or phenyl co-functionalized materials.

Soai et al. [40] have reported that highly enanselective addition of dialkylzincs to aldehydes can be achieved by using pyrrolidinylmethanols and their lithium salts. It was proposed that the lithium cation as a hard acid can easily coordinate with the oxygen atoms of benzaldehyde and proline derivative to form transition structures with less steric hindrance, leading to an enhancement in the enanometric excess of $(S)$-1-phenyl-1-propanol. Indeed, the enantiometric excess of $(S)$-1-phenyl-1-propanol is significantly increased upon addition of a small amount of $n$-butyl lithium.

\section{Conclusions}

High surface area and well-ordered mesoporous SBA-15 functionalized with chlorophropyl, methyl, and phenyl groups have been successfully prepared by co-condensation of TEOS and organic silanes in the presence of P123 as pore directing agent under strong acidic environment with the aid of $\mathrm{NaCl}$ or by grafting CPTMS on calcined SBA-15. Subsequently, the proline derivatives were anchored on the surface of SBA-15 through the nucleophilic substitution of chloropropyl groups with $(S)-(-)-\alpha, \alpha$-diphenyl-2pyrrolidinemethanol. The catalytic performances of these proline derivative SBA-15 materials were comprehensively studied during asymmetric addition of diethyl zincs to benzaldehydes. The conversion of benzaldehyde and the selectivity of (S)-1-phenyl-1propanol were found to be proportional to the loading of proline derivatives. In addition, the catalytic activities may be enhanced if the catalysts possess well-distributed and less sterically hindered proline derivatives as well as more hydrophobic surfaces. More than $80 \%$ conversion of benzaldehyde with $97 \%$ selectivity of 1 -phenyl-1propanol and $75 \%$ e.e. value could be achieved by using cat- $15 \mathrm{Cl}-$ SBA-15-t with a proline derivative loading of $0.46 \mathrm{mmol} / \mathrm{g}$ and in the presence of $n$-butyl lithium. Comparing with conventional homogeneous catalysts, the present proline-anchored SBA-15 materials that are used as chiral-selective catalysts for asymmetric addition of diethylzincs to benzaldehyde showed comparable catalytic activities and selectivities of (S)-1-phenyl-1-propanol even after repeated uses.

\section{Acknowledgments}

The financial supports from the National Science Council and from the Ministry of Education, Taiwan, are gratefully acknowledged.

\section{Appendix A. Supplementary data}

Supplementary data associated with this article can be found, in the online version, at doi:10.1016/j.apcata.2009.02.034.

\section{References}

[1] M. Tada, Y. Iwasawa, Chem. Commun. (2006) 2833-2844.

[2] S.E. Gibson, M.P. Castaldi, Chem. Commun. (2006) 3045-3062.

[3] J.M. Notestein, A. Katz, Chem. Eur. J. 12 (2006) 3954-3965.

[4] K. Soai, S. Niwa, Chem. Rev. 92 (1992) 833-856.

[5] I. Sato, T. Saito, K. Soai, Chem. Commun. (2000) 2471-2472.

[6] K. Soia, T. Shibata, H. Morioka, K. Choji, Nature 378 (1995) 767-768.

[7] L. Pu, H.B. Yu, Chem. Rev. 101 (2001) 757-824.

[8] C.E. Song, S.G. Lee, Chem. Rev. 102 (2002) 3495-3524.

[9] M. Watanabe, K. Soai, J. Chem. Soc. -Perkin Trans. I 7 (1994) 837-842.

[10] M. Watanabe, K. Soai, J. Chem. Soc. -Perkin Trans. I 21 (1994) 3125-3128.

[11] K. Hosoya, S. Tsuji, K. Yoshizako, K. Kimata, T. Araki, N. Tanaka, React. Funct. Polym. 29 (1996) 159-166.

[12] K. Soai, M. Watanabe, A. Yamamoto, J. Org. Chem. 55 (1990) 4832-4835.

[13] K. Soai, S. Niwa, H. Hori, J. Chem. Soc. Chem. Commun. 14 (1990) 982-983.

[14] C.T. Kresge, M.E. Leonowicz, W.J. Roth, J.C. Vartuli, J.S. Beck, Nature 359 (1992) 710-712.

[15] J.S. Beck, J.C. Vartuli, W.J. Roth, M.E. Leonowicz, C.T. Kresge, K.D. Schmitt, C.T.W Chu, D.H. Olson, E.W. Sheppard, S.B. McCullen, J.B. Higgins, J.L. Schlenker, J. Am. Chem. Soc. 114 (1992) 10834-10843.

[16] D. Zhao, J. Feng, Q. Huo, N. Melosh, G.H. Fredrickson, B.F. Chmelka, G.D. Stucky, Science 179 (1998) 548-553.

[17] D. Zhao, Q. Huo, J. Feng, B.F. Chmelka, G.D. Stucky, J. Am. Soc. Chem. 120 (1998) 6024-6036.

[18] J.Y. Ying, C.P. Mehnert, M.S. Wong, Angew. Chem. Inter. Ed. 38 (1999) 56-77.

[19] C.J. Brinker, Y.F. Lu, A. Sellinger, H.Y. Fan, Adv. Mater. 11 (1999) 579-585.

[20] M. Lasperas, N. Bellocq, D. Brunel, P. Moreau, Tetrahedron: Asymmetry 9 (1998) 3053-3064.

[21] D. Brunel, N. Bellocq, P. Sutra, A. Cauvel, M. Lasperas, P. Moreau, F.D. Renzo, A. Galarneau, F. Fajula, Coord. Chem. Rev. 178 (1998) 1085-1108.

[22] S. Abramson, M. Laspéras, A. Galarneau, D. Desplantier-Giscard, D. Brunel, Chem. Commun. (2000) 1773-1774

[23] S.W. Kim, S.J. Bae, T. Hyeon, B.M. Kim, Micropor. Mesopor. Mater. 44 (2001) 523 529

[24] S. Abramson, M. Laspéras, D. Brunel, Tetrahedron: Asymmetry 13 (2002) 357367

[25] A. Heckel, D. Seebach, Angew. Chem. Int. Ed. 39 (2000) 163-165.

[26] M.H. Kim, A. Stein, Chem. Mater. 11 (1999) 3285-3295.

[27] X.G. Wang, K.S.K. Lin, J.C.C. Chan, S. Cheng, J. Phys. Chem. B 109 (2005) 1763-1769.

[28] S.Y. Chen, C.Y. Tang, W.T. Chuang, J.J. Lee, Y.L. Tsai, J.C.C. Chan, C.Y. Lin, Y.C. Liu, S Cheng, Chem. Mater. 20 (2008) 3906-3916. 
[29] S.J. Huang, C.H. Huang, W.H. Chen, X. Sun, Z. Zeng, H.K. Lee, J.A. Ripmeester, C.Y. Mou, S.B. Liu, J. Phys. Chem. B 109 (2005) 681-684.

[30] M.C. Burleigh, M.A. Markowitz, E.M. Wong, J.S. Lin, B.P. Gaber, Chem. Mater. 13 (2001) 4411-4412.

[31] M.C. Burleigh, M.A. Markowitz, M.S. Spector, B.P. Gaber, J. Phys. Chem. B 105 (2001) 9935-9942.

[32] M. Llusar, G. Monros, C. Roux, J.L. Pozzo, C. Sanchez, J. Mater. Chem. 13 (2003) 2505-2514.

[33] R.M. Silverstein, F.X. Webster, Spectroscopic Identification of Organic Compounds, Wiley, New York, 1998, p. 108.

[34] S.Y. Chen, L.Y. Jang, S. Cheng, Chem. Mater. 16 (2004) 4174-4180.
[35] A. Nossov, E. Haddad, F. Guenneau, A. Galarneau, F. Di Renzo, F. Fajula, A. Gédéon, J. Phys. Chem. 107 (2003) 12456-12460.

[36] J.A. Ripmeester, C.I. Ratcliffe, Anal. Chim. Acta 283 (1993) 1103-1112.

[37] C.M. Yang, B. Zibrowius, W. Schmidt, F. Schuth, Chem. Mater. 16 (2004) 2918-2925.

[38] V.V. Terskikh, I.L. Moudrakovski, V.M. Mastikhin, J. Chem. Soc., Faraday Trans. 89 (1993) 4239-4243.

[39] S.J. Huang, S. Huh, P.S. Lo, S.H. Liu, V.S.Y. Lin, S.B. Liu, Phys. Chem. Chem. Phys. 7 (2005) 3080-3087.

[40] K. Soai, A. Ookawa, T. Kaba, K. Ogawa, J. Am. Chem. Soc. 109 (1987) 7111-7119.

[41] R. Noyori, M. Kitamura, Angew. Chem. Int. Ed. 30 (1991) 49-69.

[42] S.E. Song, J.S. Lim, K.S. Kim, K.J. Lee, D.Y. Chi, Chem. Commun. (2000) 2415-2416. 